\title{
3 O SUICÍDIO NA COMUNICAÇÃO DAS REDES SOCIAIS VIRTUAIS: REVISÃO INTEGRATIVA DA LITERATURA
}

\author{
| Camila Corrêa Matias Pereira ${ }^{1}$; Nadja Cristianne Lappann Botti²; |
}

\section{RESUMO}

INTRODUÇÃO: A Internet tem papel importante como tecnologia integradora e facilitadora aos meios de comunicação das redes sociais virtuais. As ferramentas da Internet estão presentes no avanço da globalização, integrando dimensões pessoais, sociais, comunitárias e tecnológicas e vários temas podem ser pesquisados na Internet entre eles o suicídio. O suicídio é problema de saúde pública que tem grande influência das mídias.

OBJETIVO: Identificar na literatura científica as propriedades da comunicação sobre suicídio em redes sociais virtuais.

METODOLOGIA: Foi realizada revisão integrativa da literatura através dos descritores: suicídio and rede social and comunicação. Foram definidos como critérios de inclusão artigos publicados em inglês, espanhol e português que apresentavam evidências entre a comunicação através de redes sociais virtuais (Internet) e o suicídio. Como critérios de exclusão: referências que não tinham resumo ou acesso gratuito ao texto completo on-line, artigos repetidos ou publicados em outros idiomas. Não ocorreram restrições quanto ao período de publicação, assim, foram utilizados 17 manuscritos, 16 da base de dados MEDLINE E 1 da CUMED.

RESULTADOS: A partir da leitura e análise crítica dos manuscritos emergiram duas categorias de análise: comunicação online preventiva e comunicação online prosuicida.

CONCLUSÕES: Na Internet existe fácil acesso a conteúdo sobre o suicídio tanto comunicação pró-suicida como acesso a informação, identificação, contágio e cyberbullicídio quanto comunicação preventiva como mídia preventiva, grupos de apoio online, redes sociais virtuais, telepsiquiatria. O público jovem apresenta mais vulnerabilidade pela facilidade do acesso aos meios digitais e características inerentes a adolescência.

\section{PALAVRAS-CHAVE: Suicídio; Rede social; Comunicação}

\section{RESUMEN}

"Suicidio en la comunicación de las redes sociales virtuales: Revisión integradora de la literatura"

CONTEXTO: Internet juega un papel importante como integrador y tecnología facilitadora para los medios de comunicación en redes sociales virtuales. Herramientas de Internet están presentes en la globalización, integración personal, social, comunitario, tecnológicas y varios temas se pueden buscar en Internet incluido el suicidio. El suicidio es un problema de salud pública que tiene una gran influencia de los medios de comunicación.

OBJETIVO: Identificar la evidencia de la literatura científica de las propiedades de la comunicación sobre el suicidio en las redes sociales virtuales. METODOLOGÍA: Se realizó una revisión integradora de la literatura a través de descriptores: El suicidio y la red social y la comunicación. Se definieron como criterios de inclusión los artículos publicados en Inglés, español y portugués que tenía evidencia de la comunicación a través de redes sociales virtuales y el suicidio. Criterios de exclusión: las referencias que no tenían resumen o el libre acceso al texto completo, los artículos repetidos publicados en otros idiomas. No hubo restricciones en el período de publicación, así, han usado 17 manuscritos; 16 de la base MEDLINE y 1 CUMED.

RESULTADOS: De la lectura y revisión de manuscritos emergieron dos categorías de análisis: comunicación en línea preventiva y comunicación en línea prosuicida.

CONCLUSIONES: Internet hay fácil acceso a los contenidos en el suicidio tanto la comunicación pro-suicida, el acceso a la información, identificación, contagio y cyberbullicídio, como medio de prevención de comunicación, grupos de apoyo, redes sociales virtuales, telepsiquiatria. El público joven muestra mayor vulnerabilidad para la facilidad de acceso a los medios digitales y las características de la adolescencia.

\section{DESCRIPTORES: Suicidio; Red Social; Comunicación}

\begin{abstract}
"Suicide in the communication of virtual social networks: Integrative literature review"

INTRODUCTION: The Internet plays an important role as integrator and technology facilitator to the media of virtual social networks. Internet tools are present in the advance of globalization, integrating personal, social, community and technological dimensions and various topics can be searched on the Internet including suicide. Suicide is public health problem that has great influence of the media.

AIM: Identify scientific literature evidence of the properties of communication about suicide in virtual social networks.

METHODS: It performed an integrative literature review through the descriptors: suicide and social network and communication. Were defined as inclusion criteria articles published in English, Spanish and Portuguese who had evidence of communication through virtual social networks (Internet) and suicide. Exclusion criteria: references that had no resume or free access to the full text online, or repeated articles published in other languages. There were no restrictions on the publication period thus manuscripts, so, 17 articles were used, 16 from MEDLINE's database and 1 from CUMED.

RESULTS: From reading and reviewing manuscripts emerged two categories of analysis: preventive online communication and pro suicidal online communication.

CONCLUSIONS: On the Internet there is easy access to content about suicide both pro-suicidal communication and access to information, identification, contagion and cyberbullicídio as preventive as a preventive communication media, online support groups, virtual social networks, telepsychiatry. The young audience shows more vulnerability for ease of access to digital media and adolescence inherent characteristics.
\end{abstract}

\section{KEYWORDS: Suicide; Social network; Communication}

Submetido em 13-09-2016

Aceite em 20-02-2017

1 Enfermeira, mestranda pela Universidade Federal de São João del Rei, Divinópolis, Minas Gerais, Brasil, milamatias@hotmail.com

2 Enfermeira, Psicóloga, Professora Adjunta da Universidade Federal de São João del Rei, Divinópolis, Minas Gerais, Brasil, nadjaclb@terra.com.br

Citação: Pereira, C. C.M., \& Botti, N. C. L. (2017). O suicídio na comunicação das redes sociais virtuais: revisão integrativa da literatura. Revista Portuguesa de Enfermagem de Saúde Mental (17), 17-24. 


\section{INTRODUÇÃO}

A Internet é um meio de comunicação cada vez mais presente no cotidiano das pessoas que age integrando e facilitando informações e comunicações (Secretaria de Comunicação Social da Presidência da República [SECOM], 2014). As Tecnologias de Informação e Comunicação (TICs) acompanham o mundo globalizado com diferentes intensidades de acordo com contextos sociais, políticos e culturais (Instituto Brasileiro de Geografia e Estatística [IBGE], 2015).

No Brasil, ainda é alto o percentual de pessoas que não utilizam a internet (51\%), sendo que entre os usuários que a utilizam verifica-se que acessa a internet diariamente buscando principalmente informações (76\%), diversão e entretenimento (67\%), passar o tempo livre (38\%) ou para estudo e aprendizagem (24\%). As pessoas tendem a estar conectadas geralmente nos mesmos horários durante a semana ou finais de semana. Os jovens são os usuários mais frequentes na Internet sendo que $65 \%$ das pessoas com até 25 anos e $4 \%$ das pessoas acima de 65 anos acessam diariamente. Quanto à escolaridade, $87 \%$ dos conectados com ensino superior acessam a Internet ao menos uma vez por semana e $8 \%$ daqueles que estudaram até a $4^{\text {a }}$ série fazem com a mesma frequência. As redes sociais virtuais mais utilizadas são Facebook (83\%), Whatsapp (58\%), Youtube (17\%), Instagram (12\%) e Google+ (8\%) (SECOM, 2014).

No tocante ao acesso as TICs verifica-se que o número de domicílios brasileiros com computador aumentou nos últimos anos na região Sul $(53,5 \%)$, Sudeste $(54,6 \%)$, Nordeste $(39,6 \%)$ e Norte $(35,3 \%)$. Entre os motivos para a falta de computador no domicílio encontra-se o custo elevado de equipamentos que contribui para o acesso limitador à Internet de banda larga. A banda larga está presente em $91,2 \%$ dos pacotes no Brasil enquanto os serviços de telefonia fixa totalizam $80 \%$. Nos domicílios brasileiros, 9,1\% não utiliza nenhum serviço de telecomunicação, estando na zona rural ou referem-se à população de baixa renda (Instituto de Pesquisa Econômica Aplicada [IPEA], 2014). Em 2013, identifica-se que 31,2 milhões de domicílios tinham acesso à Internet (48\% do total de domicílios permanentes), sendo que $88,4 \%$ destes utilizavam a partir de microcomputador e $11,6 \%$ por outros equipamentos. A maioria utiliza o telefone móvel ou tablet (57,3\%) para acesso à Internet. As Regiões Sudeste, Sul e Centro-Oeste $(57,0 \%, 53,5 \%, 54,3 \%$ respectivamente), registraram os maiores percentuais de utilização da Internet sem distinção de equipamentos (IBGE, 2015).
A internet tem levado a criação de novos espaços virtual e social na vida das pessoas, oferecendo-as novas perspectivas na vivência de conhecimentos bons e ruins (Kirmayer, Raikhel \& Rahimi, 2013). Entretanto, ainda pouco se tem estudado sobre sua influência na vida das pessoas (Baume, 1997). Registra-se a mídia entre os fatores de risco para o comportamento suicida. Neste aspecto verificam-se os efeitos da Internet no comportamento suicida principalmente entre os jovens devido a maior vulnerabilidade e acesso à comunicação (Thompsom, 1999).

No Brasil existem vários fóruns onde ocorrem discussões que podem ter como finalidade a discussão do suicídio sendo, então facilitada devido comunicação instantânea. Do mesmo modo, existem sites elaborados por profissionais de saúde difundindo informações preventivas para o suicídio (Gomes, Batista, Carneiro \& Cardoso, 2014).

Alguns fatores predisponentes ao fenômeno do suicídio necessitam de atenção; a fase da adolescência, a presença de eventos estressores na vida, exposição a diferentes tipos de violência, uso de drogas (lícitas e/ou ilícitas), problemas familiares, histórico familiar de suicídio, questões sociais relacionadas à pobreza, influência da mídia, questões geográficas e a depressão (Braga \& Dell'Aglio, 2013). É importante que haja maior atenção para o desenvolvimento dos programas e estratégias de prevenção do comportamento suicida e da depressão como forma de combater os fatores de risco e potencializar os fatores protetores (Erse et al., 2016). Neste contexto alerta-se para a importância de se estudar o impacto das mídias no comportamento suicida e a criação de Políticas Públicas para as comunicações em redes sociais virtuais (Gomes et al., 2014). Dessa forma o objetivo do presente estudo é identificar na literatura científica as propriedades da comunicação sobre suicídio em redes sociais virtuais.

\section{MÉTODOS}

Realizada Revisão Integrativa da Literatura que contribui para análise sistêmica de resultados visando compreender o objeto de estudo através de outros estudos independentes (Lanzoni \& Meirelles, 2011). A Revisão Integrativa da Literatura consiste na mais ampla abordagem metodológica referente às revisões, pois permite a inclusão de estudos experimentais e não-experimentais aliados a dados da literatura teórica e empírica para uma compreensão completa do fenômeno analisado (Souza, Silva \& Carvalho, 2010). 
A pergunta elaborada como questão norteadora da Revisão Integrativa da Literatura foi: Quais as evidências entre a comunicação através de redes sociais virtuais (Internet) e o suicídio?

A busca dos estudos primários foi realizada na Biblioteca Virtual em Saúde (BIREME) nas bases de dados Literatura Internacional em Ciências da Saúde (MEDLINE), Literatura Latino-Americana e do Caribe em Ciências da Saúde (LILACS) e Publicação Biomédica Cubana (CUMED). A busca foi realizada através da técnica booleana de descritores em Ciências da Saúde (DeCS) indexados, assim foram utilizados na língua portuguesa os descritores: suicídio and rede social and comunicação. Para a revisão definiu-se como critérios de inclusão artigos publicados em inglês, espanhol e português que apresentavam evidências entre a comunicação através de redes sociais virtuais (Internet) e o suicídio. Como critérios de exclusão foram eleitos: referências que não tinham resumo ou acesso gratuito ao texto completo on-line, artigos repetidos ou publicados em outros idiomas. Nesta revisão não ocorreram restrições quanto ao período de publicação.

No total foram encontrados 41 manuscritos sendo que 12 destes não tinham resumo e assim, excluídos. Dentre os 29 manuscritos restantes verifica-se que 27 são da base de dados MEDLINE, 1 da CUMED e 1 da LILACS. Posteriormente realizou-se a leitura do título seguida do resumo das 29 referências a fim de verificar se apresentavam evidências entre a comunicação através de redes sociais virtuais (Internet) e o suicídio. Após a leitura dos 29 resumos dos manuscritos, foram excluídos 12 destes sendo 1 manuscrito publicado em alemão, 9 porque a comunicação suicida não estava relacionada com a internet, 1 manuscrito que não encontrava disponível na íntegra e 1 manuscrito publicado em duas bases de dados, portanto repetido. Foram selecionados nesta Revisão o total de 17 manuscritos.

\section{RESULTADOS}

O quadro 1 mostra Informações sobre os 17 manuscritos selecionados nesta revisão.

Quadro 1 - Informações sobre os Artigos incluídos na Revisão Integrativa da Literatura, 2015.

\begin{tabular}{|c|c|c|c|c|c|}
\hline Ano & Título & Autores & Objetivo & População Alvo & Principais Conclusões \\
\hline 2004 & $\begin{array}{l}\text { Suicídio online: Os } \\
\text { sites de suicídio } \\
\text { podem desencadear } \\
\text { comportamentos sui- } \\
\text { cidas em adolescentes } \\
\text { predispostos? }\end{array}$ & $\begin{array}{l}\text { Becker, K.; } \\
\text { Mayer, M.; Na- } \\
\text { genborg, M.; } \\
\text { El-Faddagh, } \\
\text { M.; Schmidt, } \\
\text { M. H. }\end{array}$ & $\begin{array}{l}\text { Discutir se há risco de } \\
\text { suicídio para os jovens } \\
\text { vulneráveis que utilizam a } \\
\text { Internet. }\end{array}$ & $\begin{array}{l}\text { Adolescente do sexo } \\
\text { feminino de } 17 \text { anos } \\
\text { que visita o conteúdo } \\
\text { na Internet sobre os } \\
\text { fóruns sobre suicídio. }\end{array}$ & $\begin{array}{l}\text { A Internet tem riscos e benefícios. O uso e } \\
\text { abuso da Internet, a discussão sobre o suicídio } \\
\text { e as medidas legais online deveriam ser discu- } \\
\text { tidas sob perspectiva internacional. }\end{array}$ \\
\hline 2005 & $\begin{array}{l}\text { Características da } \\
\text { escrita de pessoas } \\
\text { suicidas na Internet: } \\
\text { Investigação psi- } \\
\text { cológica de ambientes } \\
\text { sociais emergentes. }\end{array}$ & $\begin{array}{l}\text { Barak, A.; } \\
\text { Miron, O. }\end{array}$ & $\begin{array}{l}\text { Analisar a diferença das es- } \\
\text { critas online de uma pessoa } \\
\text { vulnerável e apresentando } \\
\text { comportamento suicida de } \\
\text { pessoas que não estão em } \\
\text { situação de vulnerabilidade. }\end{array}$ & $\begin{array}{l}\text { Escrita online das } \\
\text { pessoas suicidas na } \\
\text { Internet. }\end{array}$ & $\begin{array}{l}\text { O comportamento online pode identificar } \\
\text { condições emocionais relacionadas ao suicí- } \\
\text { dio. A Internet pode ser uma plataforma para } \\
\text { o estudo da comunicação suicida. }\end{array}$ \\
\hline 2006 & $\begin{array}{l}\text { Suicídio no cibere- } \\
\text { spaço: Revisão sobre } \\
\text { o papel da internet no } \\
\text { suicídio. }\end{array}$ & $\begin{array}{l}\text { Alao, A. O.; } \\
\text { Soderberg, } \\
\text { M.; Pohl, E. L.; } \\
\text { Alao, A. L. }\end{array}$ & $\begin{array}{l}\text { Identificar e analisar o } \\
\text { conteúdo do suicídio na } \\
\text { Internet. }\end{array}$ & $\begin{array}{l}\text { Conteúdo publicado } \\
\text { sobre o suicídio na } \\
\text { Internet em diferentes } \\
\text { redes sociais virtuais. }\end{array}$ & $\begin{array}{l}\text { A internet é um rápido método de comunica- } \\
\text { ção mas tem benefícios e riscos. Profissionais } \\
\text { de saúde, familiares, amigos que lidam com } \\
\text { indivíduos mentalmente doentes deveriam } \\
\text { conhecer os riscos e ferramentas de prevenção } \\
\text { da Internet através da comunicação. }\end{array}$ \\
\hline 2007 & $\begin{array}{l}\text { Primeira ajuda } \\
\text { emocional em crise } \\
\text { suicida: Comparação } \\
\text { entre a linha telefônica } \\
\text { e a internet. }\end{array}$ & $\begin{array}{l}\text { Gilat, I.; Sha- } \\
\text { har, G. }\end{array}$ & $\begin{array}{l}\text { Comparar as características } \\
\text { de três serviços voluntários } \\
\text { de ajuda em relação ao suicí- } \\
\text { dio: linhas telefônicas, chats } \\
\text { e grupos online de suporte. }\end{array}$ & $\begin{array}{l}\text { Três serviços volun- } \\
\text { tários de ajuda em } \\
\text { relação ao suicídio de } \\
\text { Israel. }\end{array}$ & $\begin{array}{l}\text { As ameaças de suicídio foram mais frequentes } \\
\text { nos participantes de grupos de suporte online } \\
\text { (assíncronos) do que por telefone. Há a neces- } \\
\text { sidade de mais pesquisas sobre o fenômeno } \\
\text { do suicídio em grupos online de suporte. }\end{array}$ \\
\hline 2007 & $\begin{array}{l}\text { Questões éticas, legais } \\
\text { e práticas no controle } \\
\text { e regulação da pro- } \\
\text { moção e assistência ao } \\
\text { suicídio na Internet. }\end{array}$ & $\begin{array}{l}\text { Mishara, B. } \\
\text { L.; Weisstub, } \\
\text { D. N. }\end{array}$ & $\begin{array}{l}\text { Identificar as legislações } \\
\text { atuantes na Internet para } \\
\text { a população que apresenta } \\
\text { comportamento suicida } \\
\text { online. }\end{array}$ & $\begin{array}{l}\text { Conteúdo sobre o } \\
\text { suicídio na Internet } \\
\text { e as leis atuantes de } \\
\text { vários países sobre } \\
\text { este tipo de conteúdo } \\
\text { online. }\end{array}$ & $\begin{array}{l}\text { Há grande necessidade e é um desafio imple- } \\
\text { mentar leis atuantes na Internet para a popu- } \\
\text { lação que apresenta comportamento suicida } \\
\text { online sem ferir a liberdade de expressão. }\end{array}$ \\
\hline 2009 & $\begin{array}{l}\text { Pesquisa ética sobre o } \\
\text { suicídio: Levantamen- } \\
\text { to de pesquisadores }\end{array}$ & $\begin{array}{l}\text { Lakeman, R.; } \\
\text { Fitzgerald, M. }\end{array}$ & $\begin{array}{l}\text { Investigar formas para mel- } \\
\text { hor compreensão e resposta } \\
\text { eficaz às pessoas que são } \\
\text { suicidas. }\end{array}$ & $\begin{array}{l}\text { Pesquisadores sobre o } \\
\text { suicídio }\end{array}$ & $\begin{array}{l}\text { Os pesquisadores devem ter respeito, sensibi- } \\
\text { lidade, integridade e desenvolver relações de } \\
\text { confiança nas pesquisas com indivíduos que } \\
\text { apresentam comportamento suicida. }\end{array}$ \\
\hline
\end{tabular}




\begin{tabular}{|c|c|c|c|c|c|}
\hline 2009 & $\begin{array}{l}\text { Alerta: O lado obscuro } \\
\text { dos chats - Internet } \\
\text { sem limites. }\end{array}$ & Aderet, A. & $\begin{array}{l}\text { Investigar o conteúdo dos } \\
\text { grupos de discussão sobre } \\
\text { suicídio na Internet. }\end{array}$ & $\begin{array}{l}\text { Participantes dos gru- } \\
\text { pos de discussão sobre } \\
\text { suicídio na Internet. }\end{array}$ & $\begin{array}{l}\text { A Internet apresenta muitas facilidades, } \\
\text { mas também apresenta riscos para grupos } \\
\text { vulneráveis. O estudo mostra o risco para } \\
\text { indivíduos com comportamento suicida em } \\
\text { chats na Web. }\end{array}$ \\
\hline 2009 & $\begin{array}{l}\text { Examinando indi- } \\
\text { víduos com risco de } \\
\text { suicídio que entram } \\
\text { online para propósi- } \\
\text { tos relacionados ao } \\
\text { suicídio. }\end{array}$ & $\begin{array}{l}\text { Harris, K. M.; } \\
\text { McLean, J. P.; } \\
\text { Sheffield, J. }\end{array}$ & $\begin{array}{l}\text { Ajudar os indivíduos em } \\
\text { crise suicida, examinando as } \\
\text { pessoas em risco de suicídio } \\
\text { que fazem uso da Internet. }\end{array}$ & $\begin{array}{l}\text { Escrita online das } \\
\text { pessoas suicidas na } \\
\text { Internet. }\end{array}$ & $\begin{array}{l}\text { O comportamento online pode identificar } \\
\text { condições emocionais relacionadas ao suicí- } \\
\text { dio. A Internet pode ser uma plataforma para } \\
\text { o estudo da comunicação suicida. }\end{array}$ \\
\hline 2009 & $\begin{array}{l}\text { Estar "no limite": } \\
\text { Gerenciando o dilema } \\
\text { de ser autenticamente } \\
\text { suicida em fórum } \\
\text { online. }\end{array}$ & $\begin{array}{l}\text { Horne, J.; } \\
\text { Wiggins, S. }\end{array}$ & $\begin{array}{l}\text { Examinar como os usuários } \\
\text { de um fórum sobre suicídio } \\
\text { na Internet interagem. }\end{array}$ & $\begin{array}{l}\text { Usuários de um fórum } \\
\text { sobre suicídio na } \\
\text { Internet. }\end{array}$ & $\begin{array}{l}\text { O fórum é uma ferramenta utilizada por } \\
\text { indivíduos que apresentam comportamento } \\
\text { suicida como forma de se expressar, demon- } \\
\text { strar vulnerabilidade, buscar identificação e } \\
\text { pedir ajuda. }\end{array}$ \\
\hline 2010 & $\begin{array}{l}\text { Infraestruturas online } \\
\text { de apoio à investiga- } \\
\text { ção sobre a depressão, } \\
\text { automutilação e } \\
\text { suicídio. }\end{array}$ & $\begin{array}{l}\text { McCafferty, S.; } \\
\text { Doherty, T.; } \\
\text { Sinnott, R. O.; } \\
\text { Watt, J. }\end{array}$ & $\begin{array}{l}\text { Avaliar o modelo de segu- } \\
\text { rança online criado para a } \\
\text { identificação de indivíduos } \\
\text { em situação de risco. }\end{array}$ & $\begin{array}{l}\text { Modelo de segu- } \\
\text { rança financiado pelo } \\
\text { Conselho de Pesquisa } \\
\text { Econômica e Social da } \\
\text { Escócia. }\end{array}$ & $\begin{array}{l}\text { Modelo de segurança online criado para a } \\
\text { identificação de indivíduos com depressão, } \\
\text { comportamento suicida e de automutilação } \\
\text { obteve resultados positivos apesar de ainda } \\
\text { estar em desenvolvimento. }\end{array}$ \\
\hline 2012 & $\begin{array}{l}\text { Experiências de indi- } \\
\text { víduos ao ligar para } \\
\text { um serviço nacional } \\
\text { de prevenção do } \\
\text { suicídio: Relatório de } \\
\text { pesquisa online. }\end{array}$ & $\begin{array}{l}\text { Coveney, } \\
\text { C.M.; Pollock, } \\
\text { K.; Armstrong, } \\
\text { S.; Moore, J. }\end{array}$ & $\begin{array}{l}\text { Explorar as característi- } \\
\text { cas (auto-relatadas) dos } \\
\text { indivíduos que utilizam a } \\
\text { linha online de ajuda para a } \\
\text { prevenção do suicídio e suas } \\
\text { razões para entrar em contato } \\
\text { com o serviço e apresentar as } \\
\text { avaliações dos usuários sobre } \\
\text { o serviço recebido. }\end{array}$ & $\begin{array}{l}\text { Grupo online dos } \\
\text { Samaritanos. }\end{array}$ & $\begin{array}{l}\text { A linha de Prevenção do Suicídio (por tele- } \\
\text { fone e online) obteve grandes níveis de satisfa- } \\
\text { ção dos usuários. }\end{array}$ \\
\hline 2012 & $\begin{array}{l}\text { Mídia social e suicí- } \\
\text { dio: Perspectiva de } \\
\text { saúde pública. }\end{array}$ & $\begin{array}{l}\text { Luxton, D. } \\
\text { D.; June, J. D.; } \\
\text { Fairall, J. M. }\end{array}$ & $\begin{array}{l}\text { Investigar a influência das } \\
\text { mídias sociais no comporta- } \\
\text { mento suicida. }\end{array}$ & $\begin{array}{l}\text { Conteúdo das mídias } \\
\text { sociais. }\end{array}$ & $\begin{array}{l}\text { O estudo mostra evidências de que as redes } \\
\text { virtuais podem apresentar riscos para grupos } \\
\text { vulneráveis que fazem parte destas comuni- } \\
\text { dades. Da mesma forma, apresenta exemplos } \\
\text { de programas de prevenção, visando mostrar } \\
\text { o suicídio e a Internet na perspectiva da saúde } \\
\text { pública de forma a repensar futuras estraté- } \\
\text { gias de prevenção. }\end{array}$ \\
\hline 2012 & $\begin{array}{l}\text { Grupo de adolescentes } \\
\text { com comportamento } \\
\text { suicida e o possível } \\
\text { papel da tecnologia } \\
\text { de comunicação } \\
\text { eletrônica. }\end{array}$ & $\begin{array}{l}\text { Robertson, } \\
\text { L.; Skegg, K.; } \\
\text { Poore, M.; } \\
\text { Williams, S.; } \\
\text { Taylor, B. }\end{array}$ & $\begin{array}{l}\text { Investigar o papel das redes } \\
\text { sociais online e mensagens } \\
\text { de texto como fontes de } \\
\text { contágio após o suicídio de } \\
\text { adolescentes. }\end{array}$ & $\begin{array}{l}\text { Cluster de suicídio } \\
\text { presente em várias } \\
\text { escolas de uma cidade } \\
\text { rural da Nova Zelân- } \\
\text { dia. }\end{array}$ & $\begin{array}{l}\text { Estratégias para a identificação e manejo do } \\
\text { efeito contágio do suicídio na Internet deve- } \\
\text { riam ser criadas para orientar a população e } \\
\text { favorecer o envolvimento da sociedade em } \\
\text { geral na prevenção. }\end{array}$ \\
\hline 2012 & $\begin{array}{l}\text { Sinais de alerta para o } \\
\text { suicídio em fóruns na } \\
\text { Internet. }\end{array}$ & $\begin{array}{l}\text { McSwain, S.; } \\
\text { Lester, D.; } \\
\text { Gunn, J. F. }\end{array}$ & $\begin{array}{l}\text { Identificar se os } 10 \text { sinais de } \\
\text { alerta para sinais de risco } \\
\text { do suicídio propostos pela } \\
\text { Associação Americana de } \\
\text { Suicidologia foram identifica- } \\
\text { dos nas postagens de fóruns. }\end{array}$ & $\begin{array}{l}\text { Postagens em fóruns } \\
\text { de suicídio e fóruns de } \\
\text { automutilação. }\end{array}$ & $\begin{array}{l}\text { A Associação Americana de Suicidologia } \\
\text { criou } 10 \text { sinais de alarme para o suicídio que } \\
\text { se mostraram eficientes ao analisarem os } \\
\text { posts de fórum sobre suicídio e fórum sobre } \\
\text { automutilação. Os sinais de alarme são úteis } \\
\text { para identificar populações de risco. }\end{array}$ \\
\hline 2013 & $\begin{array}{l}\text { Telepsiquiatria: } \\
\text { Videoconferência na } \\
\text { prestação de cuidados } \\
\text { psiquiátricos. }\end{array}$ & Shore, J.H. & $\begin{array}{l}\text { Avaliar os tratamentos } \\
\text { psiquiátricos através da vid- } \\
\text { eoconferência online }\end{array}$ & $\begin{array}{l}\text { Serviço de Telep- } \\
\text { siquiatria }\end{array}$ & $\begin{array}{l}\text { Recursos de videoconferência associados à } \\
\text { psiquiatria trazem benefícios aos pacientes. }\end{array}$ \\
\hline 2013 & $\begin{array}{l}\text { Suicídio na Internet: } \\
\text { Comunidades de } \\
\text { afirmação e letalidade } \\
\text { da comunicação. }\end{array}$ & Niezen, R. & $\begin{array}{l}\text { Analisar o conteúdo dos } \\
\text { fóruns sobre suicídio na } \\
\text { Internet. }\end{array}$ & $\begin{array}{l}\text { Conteúdo publicado } \\
\text { nos fóruns sobre o } \\
\text { suicídio. }\end{array}$ & $\begin{array}{l}\text { Os fóruns podem intensificar ou diminuir as } \\
\text { tendências autodestrutivas de forma signifi- } \\
\text { cativa. A comunicação é importante na ocor- } \\
\text { rência de um suicídio, bem como nas práticas } \\
\text { terapêuticas. }\end{array}$ \\
\hline 2013 & $\begin{array}{l}\text { Respostas a uma } \\
\text { tentativa de suicídio } \\
\text { apresentada na mídia } \\
\text { social: Análise de rede } \\
\text { social. }\end{array}$ & $\begin{array}{l}\text { Fu, K. W.; } \\
\text { Cheng, Q.; } \\
\text { Wong, P. W.; } \\
\text { Yip, P. S. }\end{array}$ & $\begin{array}{l}\text { Examinar as reações escritas } \\
\text { de um grupo de microblog- } \\
\text { gers chineses expostos à } \\
\text { conteúdos de mensagens e } \\
\text { fotos de autoflagelamento. }\end{array}$ & $\begin{array}{l}\text { Grupo de microblog- } \\
\text { gers chineses. }\end{array}$ & $\begin{array}{l}\text { As comunidades online possuem benefícios e } \\
\text { riscos. Podem ter efeito contágio (incentivan- } \\
\text { do o comportamento suicida), mas também } \\
\text { podem providenciar suporte, apoio e ajuda } \\
\text { para os indivíduos vulneráveis. }\end{array}$ \\
\hline
\end{tabular}


A partir da leitura e análise crítica dos manuscritos emergiram três categorias de análise: comunicação online, comunicação online preventiva e comunicação online pró-suicida.

\section{DISCUSSÃO}

\section{Comunicação Online Preventiva}

Robertson, Skegg, Poore, Williams e Taylor (2012) investigaram o papel da Web e das mensagens de texto online no risco de suicídio entre adolescentes neozelandeses e apontam que a prevenção do suicídio, oferta de suporte e identificação de grupos de risco deve ser multiprofissional, multifatorial e intersetorial (Robertson et al., 2012). O monitoramento de sites de agências de prevenção pode antecipar ou detectar conversas de risco de suicídio auxiliando as pessoas que necessitam de ajuda (Robertson et al., 2012).

Luxton, June e Fairall (2012) em estudo de Revisão de Literatura sobre as formas que a mídia social pode influenciar o comportamento suicida ressaltam a atuação da rede virtual na prevenção do suicídio. Neste sentido observam-se: vídeos no Youtube com comunicação preventiva incluindo aqueles que oferecem serviços de ajuda; sistema de busca Google que possui ferramenta de caráter preventivo ao disponibilizar links e mensagens sobre serviços de proteção à vida; links no Facebook de programas de prevenção e números de telefones de serviços de prevenção do suicídio (como o Centro de Valorização da Vida - CVV, no Brasil, e o SOS voz amiga, em Portugal) e grupos de apoio online (Luxton et al., 2012). Pesquisa on-line com 1309 pessoas que ligaram, entre maio de 2008 e maio de 2009 , para o serviço Samaritano (serviço de prevenção do suicídio voluntário e de suporte às pessoas com problemas ou em crise) mostra como principais razões das ligações: problemas mentais, problemas familiares, términos de relacionamentos, solidão, tristeza, isolamento e abuso sexual (Coveney, 2012). Na avaliação do serviço Samaritano verifica-se evidências que os telefonemas ajudam na melhora do estado mental das pessoas que ligam, incluindo a redução de suicídio, ideação e tentativa e mais otimismo ao fim do contato telefônico (Coveney, 2012). Horne e Wiggins (2009) realizaram estudo em dois fóruns de Internet analisando durante 30 dias os posts e comentários através da psicologia discursiva. Neste sentido os autores mostram que os fóruns de suporte na internet possibilitam discussões de problemas relacionados ao suicídio.
Diferente das notas de suicídio, os fóruns na internet possibilitam análise prospectiva e retrospectiva (Horne \& Wiggins, 2009). As pessoas em fóruns de suporte de prevenção do suicídio têm maior tendência em compartilhar experiências com os membros do grupo do que os fóruns intitulados suicidas (Horne \& Wiggins, 2009).

Alao (2006) em estudo de Revisão de Literatura sobre fatores de risco e prevenção na internet a partir de casos de tentativa ou morte por suicídio nos quais a pessoas tiveram informações na Web sobre como cometer o suicídio afirma que a cada dia torna-se mais comum à participação de pessoas em grupos de suporte, autoajuda e discussão na internet facilitando à ajuda principalmente em função do anonimato para pessoas com problemas de autoconfiança, ideações paranoides ou desilusões (Alao, 2006). Em Israel, o grupo "Sahar" (nome hebreu para "suporte e escuta na internet") oferece suporte individual e de grupo, na modalidade online, e tem como pressuposto que o anonimato pode favorecer pessoas a compartilhar suas dificuldades emocionais e ideações suicidas (Alao, 2006).

O significado do anonimato na comunicação online preventiva também é verificado no estudo de Gilat e Shahar (2007) realizado durante um ano com três tipos de comunicações (chamada telefônica, chats pessoais síncronos e grupos de suporte online assíncronos) do serviço voluntário da Associação Israelita de Primeiros Socorros Emocionais. O anonimato reduz a barreira psicológica à procura de ajuda (Gilat \& Shahar, 2007). Devido à comunicação assíncrona os grupos de apoio online têm mais efetividade quando comparados com as ligações por telefone devido serem mensagens planejadas e estruturadas e chegarem a qualquer momento e a um grupo maior de pessoas (Gilat \& Shahar, 2007). Assim, a internet aparece como estratégia de prevenção do suicídio, oferecendo conhecimento profissional, ajuda e suporte através de chats, grupos e fóruns (Gilat \& Shahar, 2007).

A internet como estratégia de prevenção do suicídio também é apontada no estudo de caso realizado por Aderet (2009) do suicídio de um adolescente que obteve conhecimento de métodos em fóruns na Internet. A internet ao eliminar as barreiras de tempo e espaço e dissipar rapidamente as informações possibilita maior comunicação e relação pessoal. No entanto, o uso adequado da internet só será possível quando todos os atores (usuários, donos de sites e patrocinadores) compartilharem valores de respeito mútuo e liberdade garantindo a proteção individual (Aderet, 2009). 
Estudo exploratório de microblogs chineses de conteúdo de automutilação realizado por $\mathrm{Fu}$, Cheng, Wong e Yip (2013) aponta que as redes sociais virtuais podem ser utilizadas para identificar pessoas com risco de suicídio, sendo assim importante a construção de protocolos para serviços online. Neste caso, torna-se importante que a rede seja monitorada por profissionais de saúde e o problema do efeito da internet sobre o suicídio seja tema de interesse de pesquisadores (Fu et al., 2013). Lakeman e Fitzgerald (2009) em estudo com pesquisadores da temática do suicídio apontam que devem ser desenvolvidos protocolos de avaliação e manejo de risco de suicídio (Lakeman \& Fitzgerald, 2009). A internet como estratégia de prevenção do suicídio também é apontada no estudo de caso realizado por Shore (2013) de um paciente que fez uso do serviço de telepsiquiatria. Neste aspecto é importante a adaptação do tratamento na telepsiquiatria e desenvolvimento de legislação e de protocolos de emergência e procedimentos específicos de tratamento por videoconferência (Shore, 2013).

Importante aspecto sobre a prevenção do suicídio na Web refere-se a discussão de formas de controle. Neste sentido, Mishara e Weisstub (2007) em estudo de Revisão de Literatura sobre fatores de risco e prevenção do suicídio na internet ressaltam sobre a importância de considerações éticas sobre o controle da internet. Alguns países (Algeria, Bahreim, China, Alemanha, Irã, Coréia do Norte, Emirados Árabes e Vietnã) apresentam legislação de acesso restrito ou bloqueio de específico conteúdo na internet (Mishara \& Weisstub, 2007). Nos EUA, Inglaterra e Nova Zelândia há leis aprovadas para bloquear determinado conteúdo na internet, porém são derrubadas pela Suprema Corte devido às garantias constitucionais de liberdade de expressão. A Austrália é o único país que tem leis específicas em relação a sites que incitam o suicídio ou publiquem informações sobre métodos de perpetração do suicídio apesar de parte da população pedir mudanças na lei em detrimento da liberdade de expressão (Mishara \& Weisstub, 2007). A aprovação de leis contra a ajuda de suicídio na internet seria importante na prevenção do suicídio. Neste aspecto da questão da legislação observa-se que alguns países apresentam política de controle e restrição dos conteúdos de sites da internet sendo responsáveis pelo controle e restrição de conteúdos e permissão de utilização na Web, exceto de sites construídos fora do país (Luxton et al., 2012). Assim, campanhas públicas realizadas em conjunto com os setores da mídia, educação e saúde caracterizam-se como formas potenciais para salvar vidas (Luxton et al., 2012).
Como alternativa para a regulação encontra-se a classificação de sites usando programas de filtros para identificar e bloquear o acesso de determinados sites em computadores pessoais (Mishara \& Weisstub, 2007). Entre os importantes objetivos no controle da Internet para prevenção de suicídios é a proteção de crianças, adolescentes e pessoas vulneráveis. $\mathrm{O}$ maior sucesso alcançado até agora relaciona-se contra a pornografia infantil, porém até o momento não há iniciativas de proteção contra sites de promoção de suicídio (Mishara \& Weisstub, 2007).

Ainda em relação ao controle da promoção do suicídio outra questão difícil refere-se à jurisdição, pois mesmo que alguns países consigam controlar o conteúdo da Internet em seu país, o mesmo não pode ser feito outros países, pois muitos sites podem ser acessados de diferentes países, enviando informações e interagindo (Mishara \& Weisstub, 2007).

\section{Comunicação Online Prosuicida}

O estudo multidisciplinar sobre o papel da Web e das mensagens de texto online no risco de suicídio na adolescência na Nova Zelândia realizado por Robertson, Skegg, Poore, Williams e Taylor (2012) mostra que as comunicações eletrônicas aumentam o risco do contágio de suicídio entre pessoas jovens. Neste caso, a internet pode ser importante forma de contágio entre os que acessam ativamente websites e chat sobre suicídio. Tal consideração deve-se ao fato que vários adolescentes que morreram por suicídio tiveram acesso a este tipo de conteúdo na internet, (Robertson et al., 2012).

A ideia de contágio vem do fato de que um suicídio pode facilitar a ocorrência de outro suicídio em determinado lugar como forma de repetição. Em geral, pode ocorrer devido à comunicação de suicídio de amigos ou ícones veiculados na imprensa ou mídia, porém ressalta-se que esta influência pode ser difícil de ser identificada em curto prazo, podendo inclusive gerar casos falsopositivos (Robertson et al., 2012). Contágios também podem ser desencadeados a partir de funerais ou homenagens divulgadas na Internet, em geral quando há preocupação da idealização da morte por pessoas mais jovens (Robertson et al., 2012). Revisão de Literatura sobre a influência da rede virtual no comportamento suicida realizado por Luxton, June e Fairall (2012) revela que na Internet há informações detalhadas de métodos com descrições de formas letais e venda ilegal de remédios e; que os fóruns e chats podem ser risco para grupos vulneráveis ao influenciar decisões de suicídio devido ao encorajamento, idolatria ou pactos de suicídio (Luxton et al., 2012). 
Ressalta-se que o risco de suicídio aumenta entre pessoas vítimas de cyberbulling devido à instabilidade, isolamento e falta de esperança; sendo que quando relacionado direta ou indiretamente com o suicídio é nomeado de cyberbullicídio (Luxton et al., 2012). Ainda neste sentido, encontra-se na Revisão de Literatura de Alao (2006) que na internet as informações pró-suicida estão disponíveis em sites, chats, fóruns e grupos de discussão e, portanto, podem influenciar pessoas mais jovens; como também há perigo relacionado às farmácias online devido a receitas de medicações perigosas (Alao, 2006).

Estudo sobre as chamada telefônica, chats pessoais e grupos de suporte online do serviço da Associação Israelita de Primeiros Socorros Emocionais realizado por Gilat e Shahar (2007) mostra que a comunicação pública de material de suicídio na internet encoraja pessoas vulneráveis a considerarem cometer o suicídio.

A internet como vulnerabilidade também é apontada no estudo de caso de Aderet (2009) sobre o suicídio de um adolescente que obteve conhecimento acerca do método na Web. Neste caso a internet devido ao crescimento do número de usuários, liberdade de expressão e anonimato pode ser espaço de perigosos debates ideológicos e sociais, racismo e preconceito e assim, aumentando-se a vulnerabilidade de pessoas deprimidas (Aderet, 2009).

Estudo de microblogs chineses realizado por $\mathrm{Fu}$, Cheng, Wong e Yip (2013) aponta que a comunicação mediada por computador se caracteriza pela interação rápida entre os usuários sendo comum às mensagens relacionadas ao suicídio, respostas de preocupação, empatia, conselhos e pedidos de ajuda ou ainda cinismo e indiferença (Fu et al., 2013). Revisão de Literatura sobre fatores de risco e prevenção do suicídio na internet de Mishara e Weisstub (2007) sinaliza que o suicídio não é ilegal em muitos países e assim podem ter acesso a informações na internet. A pesquisa sobre a influência da mídia nos suicídios é concentrada na televisão e jornais revelando o aumento do risco de suicídio naqueles que tiveram contato com esse tipo de mídia.

Geralmente, quanto maior a divulgação do fato, maior é o efeito do contágio, porém não há dados empíricos sobre o risco de suicídio relacionado com a internet (Mishara \& Weisstub, 2007). De acordo com dados estatísticos há aumento do número de pessoas que morrem de suicídio seguindo as notícias da mídia de casos de suicídio, porém não é possível determinar se a morte por suicídio ocorreu por resultado de exposição da mídia ou se isso poderia ser evitado caso não houvesse tal exposição (Mishara \& Weisstub, 2007).
$\mathrm{O}$ anonimato na internet impede a possibilidade de verificar a autenticidade das informações de um site, por isso encontra-se sites que encorajam o suicídio com publicação de textos ou de informações sobre métodos com detalhes específicos (Mishara \& Weisstub, 2007) ou ainda com cartas de pessoas que suicidaram, declaração de óbitos ou fotografias de atos suicidas (Alao, 2006). Também há sites com "encorajadores do suicídio", que interagem estimulando a continuidade de planos suicidas; ou com "predadores de suicidas", que procuram pessoas que postam mensagens com características de vulnerabilidade ao comportamento suicida, incitando o suicídio e disponibilizando informações de como cometer o ato (Mishara \& Weisstub, 2007). Revisão de Literatura sobre o discurso online de sites pró-suicidas e preventivos de Niezen (2013) revela que há sites da Internet que expressam a aceitação do suicídio como boa moral ou até como dever.

O suicídio tem aumentado entre pessoas que acessam a internet, principalmente no Japão que registra várias mortes por pactos de suicídio, em geral por intoxicação por monóxido de carbono, entre pessoas que se conheceram online (Mishara \& Weisstub, 2007).

\section{CONCLUSÃO}

Este estudo de revisão teve como objetivo identificar na literatura científica as propriedades da comunicação sobre suicídio em redes sociais virtuais, sendo encontrado comunicação online preventiva e comunicação online prosuicida. Como comunicação online prosuicida verifica-se que a internet pode operar facilitando o acesso a conteúdo sobre o suicídio, informação sobre método, identificação, encorajamento, contágio e cyberbullicídio. Por outro lado, a comunicação preventiva na internet se apresenta como mídia preventiva, grupos de apoio online, redes sociais virtuais e telepsiquiatria. Ainda se verifica que o público jovem apresenta mais vulnerabilidade a comunicação online prosuicida pela facilidade do acesso aos meios digitais e característicasinerentes a adolescência, sendo assim de extrema importância aumentar a visibilidade dos sites de prevenção do suicídio.

Conclui-se que a internet relacionada ao suicídio tem relação ambígua, pois da mesma forma que existem sites direcionados para a prevenção, também existem sites que auxiliam no encorajamento ao ato suicida. Portanto, torna-se importante maior atenção por parte dos profissionais de saúde a estes sites, bem como sensibilização da população em geral para oferecer ajuda, realizar o encaminhamento para profissional de saúde mental ou denunciar o conteúdo quando necessário. 


\section{IMPLICAÇÕES PARA A PRÁTICA CLÍNICA}

Fica evidenciado a possibilidade de atuação da rede virtual como estratégia de prevenção do suicídio a partir da oferta de conhecimento profissional, ajuda e suporte através de chats, grupos e fóruns. Por isso, consideramos fundamental a capacitação dos enfermeiros para a intervenção em grupos de apoio, suporte ou autoajuda online, fóruns de suporte na internet e/ou facebook. Sugerimos a construção de protocolos para identificação de cyberbullicídio e de pessoas com risco de suicídio nas redes sociais virtuais, entretanto, ressalta-se a importância de serem construídos especificamente para serviços online (chats, grupos, fóruns, facebook, youtube, videoconferência e telepsiquiatria).

\section{REFERÊNCIAS BIBLIOGRÁFICAS}

Aderet, A. (2009). Alert: The dark side of chats - internet without boundaries. Israel Psychiatric Association, 46(3), 162-166.

Alao, A. O., Soderberg, M., Pohl, E. L., \& Alao, A. L. (2006). Cybersuicide: Review of the role of the internet on suicide. Cyberpsychology and Behavior, 9(4), 489-493.

Baume, P., Cantor, C. H., \& Rolfe, A. (1997). Cybersuicide: The role of interactive suicide notes on the Internet. Crisis, 18(2), 73-79.

Braga, L. D. L., \& Dell'Aglio, D. D. (2013). Suicídio na adolescência: fatores de risco, depressão e gênero. Contextos Clínicos, 6(1), 2-14. doi: 10.4013/ctc.2013.61.01

Coveney, C. M., Pollock, K., Armstrong, S., \& Moore, J. (2012). Callers' experiences of contacting a National Suicide Prevention helpline. Crisis, 33(6), 313-324. doi: 10.1027/0227-5910/a000151

Instituto Brasileiro de Geografia e Estatística. (2015). Pesquisa nacional por amostra de domicílios: Acesso à internet e à televisão e posse de telefone móvel celular para uso pessoal 2013.

Instituto de Pesquisa Econômica Aplicada. (2014). SIPS Sistema de Indicadores de Percepção Social: Serviços de telecomunicações.

Erse, M. P. Q. A., Simões, R. M. P., Façanha, J. D. N., Marques, L. A. F. A., Loureiro, C. L. E. C., Matos, M. E. T. S., e Santos, J. C. P. (2016). Depressão em adolescentes em meio escolar: Projeto + Contigo. Revista de Enfermagem Referência, 4(9), 37-45.

Fu, K. W., Cheng, Q., Wong, P. W. \& Yip, P. S. (2013). Responses to a self presented suicide attempt in social media. Crisis, 34(6), 406-412.
Gilat, I., \& Shahar, G. (2007). Emotional first aid for a suicide crisis: Comparison between telephonic hotline and internet. Psychiatry, 70(1), 12-18.

Gomes, J. O., Batista, M. N., Carneiro, A. M., e Cardoso, H. F. (2014). Suicídio e internet: Análise de resultados em ferramentas de busca. Psicologia \& Sociedade, 26(1), 63-73.

Horne, J., \& Wiggins, S. (2009). Doing being "on the edge": Managing the dilemma of being authentically suicidal in an online forum. Sociology of Health \& Illness, 31(2), 170184. doi: 10.1111/j.1467-9566.2008.01130

Kirmayer, L. J., Raikhel, E., \& Rahimi, S. (2013). Cultures of the Internet: Identity, community and mental health. Transcultural Psychiatry, 50(2), 165-191.

Lakeman, R., \& Fitzgerald, M. (2009). Ethical suicide research: A survey of researches. International Journal of Mental Health Nursing, 18(1), 10-17. doi: 10.1111/j.14470349.2008.00569.x

Lanzoni, G. M. M., \& Meirelles, B. H. S. (2011). Liderança do enfermeiro: Uma revisão integrativa da literatura. Revista Latino-Americana de Enfermagem, 19(3), 651-658.

Luxton, D. D., June, J. D., \& Fairall, J. M. (2012). Social media and suicide: A public health perspective. American Journal of Public Health, 102(2), 195-200.

Mishara, B. L., \& Weisstub, D. N. (2007). Ethical, legal, and practical issues in the control and regulation of suicide promotion and assistance over the internet. Suicide Life Threatening Behaviour, 37(1), 58-65.

Niezen, R. (2013). Internet suicide: Communities of affirmation and the lethality of communication. Transcultural Psychiatry, 50(2), 303-322. doi: $10.1177 / 1363461512473733$

Robertson, L., Skegg, K., Poore, M., Williams, S., \& Taylor, B. (2012). An adolescent suicide cluster and the possible role of electronic communication technology. Crisis, 33(4), 239-245. doi: 10.1027/0227-5910/a000140

Secretaria de Comunicação Social da Presidência da República. (2014). Pesquisa brasileira de mídia 2015: Hábitos de consumo de mídia pela população brasileira. Disponível em http://www.secom.gov.br/

Souza, M. T., Silva, M. D., e Carvalho, R. (2010). Revisão integrativa: O que é e como fazer. Einstein, 8(1), 102-106. Shore, J. H. (2013). Telepsychiatry: Videoconferencing in the delivery of psychiatric care. American Journal of Psychiatry, 170(3), 256-262. doi: 10.1176/appi. ajp.2012.12081064

Thompson, S. (1999). The Internet and its potential influence on suicide. Psychiatric Bulletin, 25, 449-451. doi: $10.1192 / \mathrm{pb} .23 .8 .449$ 